\title{
Kompensationslehre und denkökonomisches Prinzip
}

\author{
Von Erna Lesky, Wien
}

Die Vorstellung von der Kompensation hat sich seit mehr als einem Jahrhundert in der Kreislaufpathologie als außerordentlich fruchtbar, praktisch und dauerhaft erwiesen. Das hindert aber nicht, daß wir von ihrem Ursprung, ihrem Aus- und Umbau bedauerlich wenig wissen. In diese Lücke möchte die folgende Untersuchung vorstoßen. Sie möchte jene interessante Phase aus der Geschichte der Kompensationslehre herausgreifen, in der diese am Ende des 19. Jahrhunderts als teleologisches Dogma gebrandmarkt und in ihrer beherrschenden Stellung zumindest erschüttert wurde.

Wie konnte es geschehen, daß eine Lehre, die im teleophoben Raum der Berliner Avantgardisten einer mechanischen Analyse der Natur entstand, sich vierzig Jahre später dem Vorwurf ausgesetzt sah, eine verkappte teleologische Spekulation zu sein? Es handelt sich um die Lehre Ludwig TrauBES (1818-1876) vom Mechanismus der Zirkulationsstörungen. Als er sie in den fünfziger Jahren begründete, hat er sich nicht damit zufrieden gegeben, nur nach dem Entstehungsmechanismus der Ventildefekte und den sie begleitenden physikalischen Zeichen zu fragen. Das hatten die anatomischen Kliniker seit LAENNEC zur Genüge getan und eine Reihe von einander vielfach widersprechenden Hypothesen aufgestellt. Traube, der Pathophysiologe, hat energisch den Blick auf den Herzmuskel selbst und seine Leistungsfähigkeit gerichtet. Aber nicht allein diese experimentell zu prüfen und womöglich mathematisch exakt zu formulieren war die Aufgabe, die er sich stellte. Mit Hilfe des Tierexperimentes hoffte er endlich auch in der Herzpathologie jenen Raum zu betreten, der eine hypothesenfreie Abbildung der Wirklichkeit ermöglichte. «Erst nachdem gezeigt ist, daß nur die durch das Experiment isolierte Reihe von Bedingungen unter den vielen anderen, welche sich der Beobachtung gleichzeitig darbieten, zur Erzeugung einer bestimmten Erscheinung nöthig sei, treten wir aus dem Bereich der Möglichkeit in das gesuchte Gebiet der Wirklichkeit. „ ${ }^{1}$

Zwei Beobachtungen waren es vor allem, die sich dem Kliniker Traube bei Herzkranken gleichzeitig darboten und einer Aufklärung bedurften: Einmal gab es Herzen mit beträchtlicher Veränderung des Klappenapparates, ohne daß die davon Betroffenen ein Krankheitszeichen, sei es Dyspnoe,

1 Traube (1846) IV. 
Zyanose oder Asthma, aufwiesen; vielmehr fühlten sie sich trotz der Schadhaftigkeit ihres Klappenapparates relativ wohl. Zum andern aber ließ sich bei ebendenselben Fällen feststellen, daß das Herz in einzelnen Abschnitten hypertrophiert war. Der Schluß lag nahe, die Herzhypertrophie als kompensatorische Einrichtung zu deuten und durch sie das relative Wohlbefinden solcher Patienten zu erklären. Traube hat diesen Schluß gezogen, indem er annahm ${ }^{2}$, daß «jeder Klappenfehler die Leistungsfähigkeit des Herzmuskels (verringert), wodurch eine Erhöhung der Spannung im Venensystem und eine Verringerung der Spannung im arteriellen Gefäßsystem gesetzt wird. Eine Hypertrophie der Ventrikel, die sich hinzugesellt, ist nun imstande, die Leistungsfähigkeit derselben zu vermehren und genannte Spannungsveränderungen wieder auszugleichen. Es compensiert also eine Hypertrophie der Ventrikel die Fehler, welche durch den Klappenfehler hervorgerufen wurden.»

Als der Experimentalpathologe, Samuel von Basch $(1837-1905)^{3}$, der eine ausgezeichnete experimentelle Schulung im Wiener und später im Leipziger Laboratorium CARL LUdwigs empfangen hatte, sich intensiv mit Kreislaufproblemen zu beschäftigen begann, ging er dabei von der Auseinandersetzung mit der Traubeschen Kompensationslehre aus. Einmal war sie am Anfang der achtziger Jahre noch immer die herrschende. Zum andern aber entsprach es entschieden dem Forschernaturell Baschs, sich durch die Kritik an bestehenden Lehren den Weg zu neuen Erkenntnissen zu eröffnen. Er gehörte also jenem zweiten Typ von Entdeckern an, den E.H.ACKenkneGhт ${ }^{4}$ als den vornehmlich "kritischen» hervorhob, indem er diesen Typ jenem andern gegenüberstellte, der wenig Interesse an Polemik zeigt, sondern lieber in noch unbebautes Neuland vorstößt.

Was Basch an der Traubeschen Kompensationslehre kritisiert, ist nicht der Umstand, daß sie die Herzhypertrophie als direkte Folge des bestehenden Klappenfehlers erklärt, vielmehr, daß sie darüber hinaus noch einen zweiten Kausalzusammenhang postuliert, nämlich jenen «zwischen der Hypertrophie des mit einem Klappenfehler behafteten Herzens und dem Wohlbefinden seines Inhabers $\gg .^{5}$ Erst wenn auch dieser Zusammenhang auf streng induktivem, d.h. experimentellem Wege erwiesen wäre, sei man

2 Traube (1878) 70.

3 Vgl. Lesky (1965) 558 ff. Dort weitere Literatur zu Basch.

4 Ackerknecht (1957) 49.

5 Basch (1893) 258. 
berechtigt, die Herzhypertrophie als eine kompensatorische Einrichtung zu deklarieren. Solange dies aber nicht der Fall sei, kommt nach Basch der Kompensationslehre «höchstens der Werth einer vielleicht plausiblen, aber nicht gestützten Hypothese $»^{6} \mathrm{zu}$.

Es war geradezu unerhört, Traube, dem streng und bedächtig schließenden Schüler Sкораs, dem Schöpfer der experimentellen Pathologie in Deutschland, den Vorwurf zu machen, daß seine Beweise für die kompensatorische Funktion der Herzhypertrophie «nicht direct aus den Thatsachen entspringen $"{ }^{7}$ sondern sich nur an Tatsachen anlehnen. Ein solcher Vorwurf traf zentral den Kernsatz des Traubeschen Programms ${ }^{8}$ : "Aus einer gründlichen Kenntnis der bereits gewonnen Thatsachen ... hervorgegangene, scharf gestellte Fragen nach den Bedingungen der wahrgenommenen Erscheinungen und eben so bestimmte Antworten, welche das Resultat einer systematischen Reihe genauer Versuche, und zwar unmittelbarer Folgerungen aus diesen Versuchen sind: dies sind die unerläßlichen Bedingungen einer experimentell-pathologischen Arbeit.»

Überprüft man die Beweisführung Traubes für den kompensatorischen Wert der Herzhypertrophie - sie ist in seiner epochemachenden, 1856 erschienenen Arbeit «Über den Zusammenhang von Herz- und Nieren-Krankheiten ${ }^{9}$ niedergelegt -, so muß man allerdings Basch zugeben, daß die so zuversichtlich als «Werth» angesprochene Erscheinung der Kompensation sich nicht als Resultat «unmittelbarer Folgerungen» aus Versuchen darbietet. Schon die eingestreuten «offenbar» und «zweifellos» lassen erkennen, daß es sich hier nicht um ein reales Versuchsergebnis, sondern nur um ein Gedankenexperiment handelt. Sicherlich arbeitet Traube in ihm mit physikalischen Größen, wenn er die Beschleunigung $G$ der Blutmasse $M$, die diese durch die Triebkraft des Ventrikels $K$ erfährt, durch die Gleichung $K / M=G$ ausdrückt. Sicherlich hat er auch die Widerstände bei der Entleerung des Ventrikels, den Flächeninhalt seiner Ausmündung, das Volu-

${ }^{6}$ Ebenda. Hiefürist ein Satz Machs über die Hypothesenbildung charakteristisch, den Basch aus dessen Prager Rektoratsrede von 1883 «Über Umbildung im naturwissenschaftlichen Denken » (Mach 1910, 259) der Arbeit von 1893 als Motto voransetzte. Er illustriert aufs beste Baschs eigene Überzeugung in bezug auf die Kompensationslehre: «Hypothesen werden auch später erst nachteilig und dem Fortschritte gefährlich, sobald man ihnen mehr traut als den Tatsachen selbst und ihren Inhalt für realer hält als diese, sobald man, dieselben starr festhaltend, die erworbenen Gedanken gegen die noch zu erwerbenden überschätzt.»

7 Basch (1893) 260.

8 Traube (1846) V f. 
men der mit der Systole entleerten Blutmenge, die Spannung des Arterienrohrs, ja sogar das spezifische Gewicht des Blutes in Rechnung gesetzt. Aber tatsächlich messen konnte er mit den Mitteln seiner Zeit, mit dem Ludwigschen Hämodynamometer, nur die Druckverhältnisse im Aortensystem. Die gleichzeitig vor sich gehenden Druckveränderungen im rechten Ventrikel, in den beiden Vorhöfen und in den beiden Venensystemen waren unberücksichtigt geblieben.

So konnte Basch gegen Traube den Vorwurf erheben, daß er sich bei seinem hämodynamischen Raisonnement «mit einem allgemeinen, im Ganzen und Großen richtigen Satze,${ }^{10}$ nämlich dem von der Verlangsamung des Blutstroms bei Herzfehlern, zufriedengab und daß seine Kompensationslehre genauso allgemein blieb wie der Satz, aus dem er sie abgeleitet hatte. Wie lange diese generalisierende und schematisierende Tendenz der klinischen Kompensationslehre noch anhing, kann man bei dem Berliner Kliniker Friedrich Kraus (1858-1936) nachlesen ${ }^{11}$, der noch 1919 kritisch bemerkt: «Aber auch heute wird immer noch viel zu einseitig mit Blutdrucksenkung und Blutdruckverlangsamung ... operiert. Die Vitien werden hinsichtlich ihrer direkten Folgezustände viel zu sehr gleichgestellt.» Oder, um wieder mit Basch zu sprechen ${ }^{12}$ : «... sie alle insgesammt (werden) von dem schirmenden Dach der Compensationshypertrophie bedeckt.»

So interessant und nachhaltig der medizinische Teil der Streitfrage sein mag, ihr volles Gewicht und ihren für die Zeit typischen Aspekt erhält sie doch erst durch ihren geistesgeschichtlichen oder genauer durch ihren erkenntnistheoretischen Hintergrund. Basch hat seine Polemik gegen Traube am 13. Kongreß für Innere Medizin 1894 in München vorgetragen und ihr den bezeichnenden Titel «Die Compensationslehre vom erkenntnistheoretischen Standpunkte ${ }^{13}$ gegeben. Hier stellt er zwei Wege naturwissen-

9 Traube (1871) 2, $304 \mathrm{ff}$.

10 Basch (1893) 276.

11 Kraus Friedrich, Die allgemeine und spezielle Pathologie der Person, Klinische Syzygiologie, Allgemeiner Teil, Leipzig 1919, S.20. Vgl. ferner derselbe, Art. «Myokarderkrankungen », Real-Encyclopädie der gesamten Heilkunde, herausgegeben von A. EulenBurG, 4. Auflage, Band 10, Berlin/Wien 1911, S. 126.

12 Basch (1893) 277.

13 Zit.im Schrifttum. Es hängt wohl mit der Verehrung, die Basch für Traube fühlte, zusammen, daß er in dieser Rede nur von der Kompensationslehre spricht, ohne den Namen Traubes zu nennen. Dieser wird ausdrücklich genannt in den polemischen Stellen der Arbeiten (1891) 175, (1892) 158f., (1893) 259f. 276f. u.ö. 
schaftlicher Forschung einander gegenüber: den einen, der in einem ungezügelten Kausalitätsbedürfnis danach strebt, dem daraus entspringenden Gedanken objektive Tatsachen gewaltsam unterzuordnen, und den zweiten, der sich bemüht, den Tatsachen zwanglos die Gedanken anzupassen. Traube muß es sich mit seiner Kompensationslehre gefallen lassen, als Schulbeispiel für den ersten Weg angeführt zu werden. Ausgehend von der Diskrepanz zwischen objektiv nachweisbarem Befund (Herzklappenfehler) und subjektiver Beschwerdefreiheit (Fehlen von Dyspnoe, Zyanose und Stauungserscheinungen) habe Traube an Stelle der Beschreibung jener Vorgänge, die diese Phase des Krankheitsprozesses charakterisieren, die Erklärung, d.i. den Gedanken der Kompensation, gesetzt. Ausgehend von der Frage: Warum geht es Herzkranken gut, denen es von Natur schlecht gehen müßte? sei er verleitet worden, einen tatsächlichen Folgezustand wie beispielsweise die Hypertrophie des rechten Ventrikels bei Mitralinsuffizienz zur Bedeutung einer Ursache, und zwar einer bessernden, kompensierenden zu erheben. Derart Folge und Ursache vertauschend, sei er zur Annahme gekommen, die Hypertrophie des rechten Ventrikels schütze solche Kranke vor den Folgen der Stauung im Lungenkreislauf, dem Auftreten von Atemnot, indem sie die Verlangsamung des Blutstroms, weiter die Störung des Lungengasaustausches und die ihr folgende Erregung des Atemzentrums verhindere. Man sieht: die Debatte geht letztlich um die Bedingungen, unter denen Dyspnoe entsteht bzw. nach Traubes Kompensationslehre verhindert wird. Man sieht ferner, daß Traube die Dyspnoe vornehmlich als eine Störung neurochemischer Natur (Lungengasaustausch - Atemzentrum) aufgefaßt haben wollte.

$\mathrm{Zu}$ einer anders akzentuierten Auffassung ist Basch auf einem anderen Wege gelangt, den er selbst als den zweiten Weg, den Weg der zwanglosen Anpassung der Gedanken an die Tatsachen, verstanden wissen will. Allerdings standen ihm und seinen Schülern dabei die Methoden einer inzwischen verfeinerten Experimentaltechnik zur Verfügung. Basch und seine Schüler ${ }^{14}$ haben bei der Mitralinsuffizienz nicht nur die erhöhte Spannung in der Pulmonalarterie gemessen, wie es schon Traube tat; sie konnten auch die Spannung im linken Vorhofe messen und feststellen, daß sie zugleich mit der Spannung in der Pulmonalarterie steige, da 3 also das Blut unter niedrigerem Gefälle, aber hohem Drucke in den stark gespannten und dement-

14 Die Versuchsergebnisse aus Baschs Laboratorium finden sich in den im Schrifttum zitierten Klinischen und experimentellen Studien im einzelnen dargestellt. 
sprechend erweiterten Lungenkapillaren fließe. Indem Basch so Schritt für Schritt die durch den Klappenfehler gesetzten Bedingungen isolierte und analysierte und den neuen Tatsachen seine Gedanken anpaßte, gelangte er zu dem Begriff der Lungenschwellung und Lungenstarre ${ }^{15}$, d.h. zu der Auffassung, daß die Lunge durch die Überfüllung ihrer Gefäße in einen Zustand der Erektion gerät, der notwendigerweise ihre Atmungsfähigkeit beeinträchtigt. Mit dieser Auffassung hat Basch ein neues Moment in die Erklärung der Dyspnoe eingeführt. $\mathrm{Da} ß$ diese nicht nur neurochemisch, sondern auch mechanisch durch die Verkleinerung der Ventilationsgröße der Lunge bedingt sei, ist seither als gesichertes Wissen in die übrigens noch in so vielem hypothetische Lehre von der kardialen Dyspnoe eingegangen ${ }^{16}$.

Man brauchte auf der ersten Seite des gedruckten Münchener Vortrages nicht den Namen Mach in gesperrten Lettern und den Hinweis auf seine Rede zu lesen, die er «Über das Prinzip der Vergleichung in der Physik» auf der Wiener Naturforscherversammlung des Vorjahres $1894^{17}$ gehalten hat. Allein die auf den ersten Seiten gebrauchte Terminologie, «Beziehung der Bedingung zum Bedingten», "Anpassung der Gedanken an die Tatsachen », "Auflösung des Erscheinungskomplexes in seine Elemente», die in der Folge am Beispiel der Mitralinsuffizienz durchexerziert wird, macht

15 Die erste Publikation darüber Wien.med.Blätter 10 (1887) 465-467, die zweite «Die cardiale Dyspnoe und das cardiale Asthma ", Klin.Zeit- Streitfragen, herausgegeben von J.Schnitzler, Band 1, Heft 3 und 4, Wien 1887. Zentral der Vortrag «Über Lungenschwellung und Lungenstarrheit», Wien.med.Presse 29 (1888), 587-594= Basch (1891) $171 \mathrm{ff}$. Vgl. ferner ebenda S. $183 \mathrm{ff} .191 \mathrm{ff}$., $198 \mathrm{ff}$. Zur Kritik, die hier nicht mehr einbezogen werden kann, vgl. die Stellungnahmen von A. Fraenkel, Über cardiale Dyspnoe, Berliner klin.Wschr. 25 (1888) 289-292, 315-317. Derselbe, Diagnostik und allgemeine Symptomatologie der Lungenkrankheiten, Wien/Leipzig 1890, S. 185 f. (scharf ablehnend); H.v.BAMBERGER, Wiener klin. Ws schr. 1 (1888) $90 \mathrm{ff}$. = Basch (1891) 1 , $183 \mathrm{ff}$. (ablehnend); O. KaHLER, Wiener klin. Wschr. 1 (1888) $227 \mathrm{f}$. (ablehnend); O. RosenBaCH, Die Krankheiten des Herzens und ihre Behandlung, Wien/Leipzig 1897, S. $386 \mathrm{ff} . ;$ derselbe, Grundriß der Pathologie und Therapie der Herzkrankheiten, Berlin/Wien 1899, S.185 (scharf ablehnend); L.KrenL, Pathologische Physiologie, 2.Auflage, Leipzig 1898, S.48f. (zustimmend); derselbe, Die Erkrankungen des Herzmuskels, Wien 1901, S. 112 (ablehnend); W.v.Leube, Specielle Diagnose der inneren Krankheiten, Band 1, Leipzig 1904, S. $90 \mathrm{ff}$. (zustimmend). Über die spätere Entwicklung der Frage vgl. A.Strubell-Harkort, Vasomotorische Einflüsse und Druckverhältnisse im kleinen Kreislauf, Verhandlungen der Deutschen Gesellschaft für Kreislaufforschung, 8. Tagung, Dresden/Leipzig 1935, S. $129 \mathrm{ff}$.

16 E. Lauda, Lehrbuch der inneren Medizin, Band 1, Wien 1949, S.37, 337.

17 Wiederabgedruckt in Mach (1910) 266-289. 
sofort offenbar, daß sich Basch hier in der ureigensten Gedankenwelt seines Freundes aus der Wiener Studienzeit, des späteren Prager Physikprofessors Ernst Mach (1838-1916) ${ }^{18}$ bewegt, der eben 1895 als Professor der Philosophie nach Wien zurückkehrte. Von der außerordentlichen Wirkung, die Machs Erkenntnislehre auf das Denken in der Medizin seiner Zeit ausübte, ist bisher ebenso wie über seine reichen Mediziner-Kontakte nur das Wenigste bekannt. Schon in den frühen sechziger Jahren hat Mach sich in den Laboratorien der Wiener Physiologen Ernst Wilhelm von BrücKe und CARL Ludwig ${ }^{18 a}$, in denen er außer Basch auch Josef Breuer begegnete, die Probleme der Mediziner zu seinen eigenen gemacht. So ist es nicht nur ein Zufall, daß er fast gleichzeitig mit Breuer, wenn auch unabhängig von ihm, 1873 seine Strömungstheorie der Endolymphe entwickelte ${ }^{19}$. Die gemeinsame, auf die mechanische Analyse der Natur gerichtete Seh- und Arbeitsweise hat in allen diesen Brücke- und Ludwig-Schülern in gleichem Sinne fortgewirkt. Kein Wunder, daß sie und unter ihnen Basch 1895, als Mach sein denkökonomisches Prinzip voll ausgebaut hatte, begierig nach diesem als der ihrer Schule gemäßen Erkenntnislehre griffen. Freilich ist es nicht so, daß Basch seine neuen Erkenntnisse in der Kreislaufpathologie nach dem Rezept der Machschen Denkökonomie gewonnen hätte. Auch hier gilt der Satz Claude Bernards ${ }^{20}$ : «daß die Verfahren und Methoden der Wissenschaft sich nur in den Laboratorien erlernen lassen.» Aber sich retrospektiv über die Struktur des Erkenntnisvorganges Rechenschaft abzulegen, war Basch ebenso Bedürfnis, wie es das für Helmholtz, Virchow und Du Bors-ReYmond gewesen ist.

So hat er denn seine Auseinandersetzung mit der Kompensationslehre Traubes als Beispiel dafür entwickelt, daß die Machsche Grundantithese von Beschreibung: Erklärung nicht nur im anorganischen, sondern auch im organisch-biologischen Bereich sich als gültig erweist. Mit den Worten ${ }^{21}$ «wir können nicht erklären », nimmt er das Leitmotiv Machs als methodologisches Postulat auf und setzt es in dessen agnostizistischem Sinn fort: «... noch weniger sind wir im Stande, den Beweis für die letzten Ursachen

18 Die wichtigste Literatur zu Mach ebenso wie seine Werke findet man aufgeführt bei J.THiELE, Ernst-Mach-Bibliographie, Centaurus 8 (1963) 189-237.

${ }^{18 a}$ Wie enge die Beziehungen Machs zu Ludwig gewesen sind, enthüllen Briefe Ludwigs an Mach, über die demnächst in der Clio Medica gehandelt werden wird.

19 Dazu vgl. Lesky (1965) 534f. mit weiterer Literatur.

20 Cl. Bernard, Einführung in das Studium der experimentellen Medizin, Paris 1865, Sudhoffs Klassiker der Medizin, Band 35, Leipzig 1961, S.313. 
zu führen, deren Ergründung unser Causalitätsbedürfnis ... gierig nachstrebt.» Indem Traube sich von diesem Kausalitätsbedürfnis hinreißen ließ, die Frage nach dem Warum an Stelle jener nach dem Wie in den Vordergrund zu rücken, habe er eine Reihe von Tatsachen, die sich anders verknüpfen ließen, unter den Kompensationsgedanken gezwungen und damit eine neue Ontologie begründet.

«Wir können nur die Beziehungen kennenlernen, welche zwischen der bedingten sinnlichen Erscheinung und der sie bedingenden, gleichfalls sinnfälligen bestehen.» Mit diesem Satz kennzeichnet Basch seinen eigenen Forschungsweg im Sinne Machs als eine «Beschreibung» funktioneller Beziehungen. Die mit dem Manometer gemessene Drucksteigerung im linken Vorhof, die perkutorisch nachweisbare Vergrößerung der geschwellten und starren Lunge und die Vergrößerung des hypertrophen rechten Ventrikels boten sich zunächst quantitativ und qualitativ als verschiedene Erscheinungen den Sinnen dar. Indem Basch sie aber alle durch die Bedingung der Mitralinsuffizienz als bedingte Erscheinungen experimentell nachwies, erfüllte er das Machsche Postulat der «Beschreibung», d.h. den Bereich der Phainomena nirgends zu überschreiten. Von dieser Position - es ist die ge-

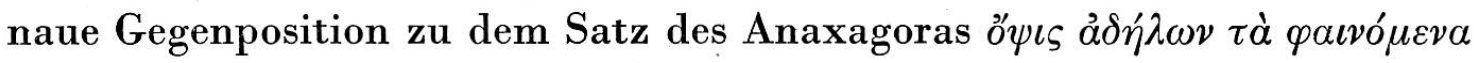
- gelangte er auch zur strikten Ablehnung von Traubes Kompensationslehre. So «mußte nothgedrungen das hypertrophische rechte Herz um seinen Ruhm als Wohlthäter, als Compensator der Klappenfehler des linken venösen Ostiums kommen ».22

Bei dieser Ablehnung der Kompensationslehre hat sich Basch in Übereinstimmung mit einem weiteren Machschen Denkprinzip gefühlt: mit der Anpassung der Gedanken an die Tatsachen. Mach selbst hat in seinen «Leitgedanken » ${ }^{23}$ dieses Prinzip schlechthin «als Aufgabe der wissenschaftlichen Erkenntnis» bezeichnet und es in engsten Zusammenhang mit dem darwinistischen Ideengut gebracht. Wenn er die eine Wurzel seines denkökonomischen Prinzips - denn um dieses handelt es sich - vom « Überleben des Passendsten » im «Wettstreit der wissenschaftlichen Gedanken» herleitet, so ist es wohlbegründet, daß er diese Wurzel als «biologische» bezeichnete, und ebenso, daß er sie mit der von ihm geforderten Ökonomie

21 Basch (1895) 433.

22 Basch (1893) 277.

23 Mach (1919) 3f. Vgl. ferner F. Kallfelz, Das Ökonomieprinzip bei Ernst Mach, Phil. Diss., München 1929. Heller (1964) $48 \mathrm{ff}$. 
des Denkens in seinem Satze vereinte. Denn: «Diese Ansicht (sc. die biologische) widerspricht nicht der ökonomischen Auffassung, sondern läßt sich, diese ergänzend, mit ihr zu einer biologisch-ökonomischen Darstellung der Erkenntnislehre vereinigen.»

So ist es denn Mach, von dem unmittelbar der Begriff der Anpassung in den erkenntniskritischen Teil der Münchener Rede einströmte. Der Anpassungsbegriff beherrscht aber ebenso auch ihre anderen Zusammenhänge, ihre physiologischen und pathologischen Analysen. Ja, in diesen wird er geradezu zum Kristallisationskern, um den herum sich Baschs eigenste Synthese des Kreislaufgeschehens formiert. Auf welchen Wegen kommt nun dieser Anpassungsbegriff Basch zu?

Wir können hier nur das Wesentliche hervorheben, wenn wir feststellen: Es hat immerhin 22 Jahre gebraucht, bis Wilhelm Roux, der Begründer der Entwicklungsmechanik, nach dem Erscheinen von On the origin of species (1859) in seiner durch den Titel allein schon als darwinistisch signierten Monographie Der züchtende Kampf der Theile (1881) den Begriff «funktionelle Anpassung» für die mechanische Entstehung des Zweckmäßigen im Sinne des «Dauerfähigen» prägte. Sofort hat das neue Leitwort Anatomen, Physiologen, Pathologen und Kliniker fasziniert und eine mächtige Welle von Versuchen ausgelöst, um im Morphologischen und Funktionellen seine Leistungsfähigkeit zu erproben ${ }^{24}$. In der Tat waren eine Reihe von bislang unverständlichen Erscheinungen, wie die Architektonik der Knochenbälkchen, nunmehr verstehbar, eine Fülle anderer, wie das Anpassungsvermögen des Herzmuskels an erhöhte Anforderungen, überhaupt erst entdeckt worden. Den experimentellen Beweis hiefür hatten die Versuche von Rosenbach, Cohnheim und Lichtheim erbracht. Aber sie waren in der Deutung der Anpassung auf dem Boden der Traubeschen Kompensationslehre geblieben. Erst Basch verläßt ihn auch hier, und zwar genau an jenem Punkt, von dem seine Kritik an Traube ihren Ausgang genommen hatte: am Widerspruch zwischen relativer Beschwerdefreiheit und objektivem Befund bei Herzklappenfehlern. Indem er das Ausbleiben der Atemnot als eine Anpassungserscheinung nicht erklärt, sondern «beschreibt», fühlt er sich auch hier als Verwirklicher des Machschen methodologischen Postulats ${ }^{25}$ : «Indem wir das Walten dieser Fähigkeit (sc. der Anpassung) ...

24 Vgl. H. NothNageL, Die Anpassung des Organismus bei pathologischen Veränderungen, Wiener klin. Wschr. 7 (1894) 282-284.

${ }^{25}$ Basch (1895) 440. 
auch hier erkennen, verstehen wir, daß die Reaktion der Athemnot nicht nur ausbleibt, wenn die sie veranlassenden Bedingungen eine bestimmte Größe noch nicht erreicht haben, wir verstehen auch, daß sie ausbleibt, trotzdem deutliche objektive Merkmale zu erkennen geben, daß diese Entstehungsbedingungen schon einen hohen Grad von Ausbildung erreicht haben.» Selbst im Stadium der ausgebildeten Lungenschwellung und Rechtshypertrophie «(bleibt) das Ausbleiben der Athemnot noch immer als eine Anpassungserscheinung verständlich». In diesem Stadium vollends wird der Gegensatz zwischen Baschs Akkomodationshypertrophie als einer rein mechanischen Gesetzen folgenden Anpassungserscheinung und der Kompensationshypertrophie Traubes als einer teleologisch belasteten Hypothese in aller Schärfe offenbar. Von dieser unterscheidet sich jene schon allein durch den Namen «Akkomodationshypertrophie», noch mehr aber durch die beigegebenen negativen Attribute. Denn die Akkomodationshypertrophie kann in der Sicht von Basch weder eine Atemnot verhindern, noch den gehemmten Blutstrom beschleunigen oder gar seinen Druck erhöhen. Vor der letzten Möglichkeit müßte man sogar erschrecken. Denn wenn sie in der Tat so etwas vermöchte, würde sie mit der Drucksteigerung in der Lunge nur die Lungenschwellung vermehren und so den Herzfehler verschlechtern. Weit entfernt, sich wie die Kompensationshypertrophie als Wohltäterin der Klappenfehler zu gebärden, ist Baschs Akkomodationshypertrophie schon zufrieden, wenn sie mit ihrer stärkeren Herzarbeit kein Unheil anrichtet und den Status quo erhält. Weiter will sie nichts, und damit ist im teleologie-freien Raum der Machschen Erkenntnislehre für Basch das Problem der Kreislaufstörungen im Sinne von Akkomodationsstörungen vom wissenschaftlichen Standpunkt aus gelöst.

Hie Akkomodationshypertrophie bzw. Akkomodationsstörungen - dort Kompensationshypertrophie bzw. Kompensationsstörungen: mit dieser Terminologie sind die Positionen in der Deutung des Kreislaufgeschehens am Ende des Jahrhunderts bzw. seiner Mitte klar bezeichnet. Es wäre gefehlt, sie als einen müßigen Nomenklaturstreit abzutun. Basch hat sich ausdrücklich dagegen verwahrt ${ }^{26}$. Denn was sich hinter diesem Namen verbirgt, ist mehr als eine Nomenklaturverschiebung, ist eine echte Sinnveränderung, wie sie der Einbruch der Begriffswelt des Darwinismus und des Neopositivismus Machscher Prägung im medizinischen Denken heraufführte. Nach der Rezeption dieser Ideenwelt mußte der Mechanist von

26 Basch (1892) 160, Anmerkung. 
Gestern, Traube, der 1846 ausgezogen war, um eine hypothesen- und zweckfreie Abbildung der Wirklichkeit zu begründen, dem Mechanisten der neunziger Jahre, Basch, als ein teleologischer Spekulant erscheinen. So sehr war Basch in der Aneignung der neopositivistischen Erkenntnislehre methodologisch empfindlich geworden, daß ihm schon allein der teleologische Ansatz, den nun einmal die Traubesche Kompensationslehre darbot, nachgerade unerträglich wurde.

Wäre es nach Basch gegangen, so hätte er in seinem darwinistisch-neopositivistischen Supermechanismus alles Lebendige in den Röhren, Schläuchen und Ventilen seines Kreislaufmodells ${ }^{27}$ mit Siegellack und Gummi eingefangen und mit Quecksilber- und Sodamanometer gemessen. Daß er wenigstens die Reservekraft des Herzens mit seiner Maschine messen konnte ${ }^{28}$, hat ihm besondere Freude bereitet, weil sie ja nach Ansicht der Teleologen etwas Unmeßbares, geheimnisvoll Lebendiges war. Er mag es aber auch als eine besondere Ironie empfunden haben, daß gerade im Zusammenhang mit dem Anpassungsbegriff die so energisch vertriebene Teleologie noch zu seiner Lebenszeit durch eine Hintertüre wieder hereinkam. Schon in den neunziger Jahren begannen sich vitalistisch-teleologische Deutungen wieder zu regen ${ }^{29}$. Das neue Jahrhundert öffnete ihnen breit das Tor. Die alte, alles vermögende Lebenskraft der Romantiker erlebte in mannigfachen $\mathrm{Ab}$ wandlungen als Entelechie (Driesch), Dominanten- und Systemkräfte (REINKe), als Impulssysteme (UEXKüLL) oder gar als Atomseele (R.Косн) eine kräftige Wiedergeburt ${ }^{30}$. In wieder anderer Form bietet sich dem Heutigen der uralte Gegensatz zwischen mechanistischer und teleologischer Welterklärung dar. Und so sind die hier in schmalem Ausschnitt behandelten Fragen bis heute nicht zur Ruhe gekommen, sondern perennierende geblieben. Vielleicht deshalb, weil «der Mensch bei dem Glauben verharren (muß), daß das Unbegreifliche begreiflich sei, er würde sonst nicht mehr forschen ».

27 Abgebildet ebenda S.7, Fig. 1. Dazu vgl. man die Kritik O. Rosenbachs, Grundriß der Pathologie und Therapie der Herzkrankheiten, Berlin/Wien 1899, S. $180 \mathrm{ff}$., der vom Standpunkt seines energetischen Funktionalismus aus den mechanistischen Baschs scharf ablehnt.

${ }^{28}$ Basch (1892) 11.

29 Vgl. P.Diepgen, Mechanismus und Vitalismus in der zweiten Hälfte des 19. Jahrhunderts, Münchner med.Wschr.96 (1954) 203-205.

30 E. Albrecht, Vorfragen der Biologie, Wiesbaden 1899, S. 11 ff. B. Fischer, Vitalismus und Pathologie, Berlin 1924, S.2f. 


\section{Schrifttum}

Ackerknecht Erwin Heinz, (1957) Rudolf Virchow, Arzt, Politiker, Anthropologe, Stuttgart.

Basch Samuel von (1891), Klinische und experimentelle Studien, Band 1, Beiträge zur Pathologie des Kreislaufs, Berlin.

- (1892), Allgemeine Physiologie und Pathologie, Wien.

- (1893), Historisches und Kritisches zur Lehre von der Compensation der Herzfehler, Wien.klin.Wschr.6 (1893) 257-261, 274-278.

- (1895), Die Compensationslehre vom erkenntnis-theoretischen Standpunkte. Verhandlungen des 13. Congresses für Innere Medicin, Wiesbaden, S.433-447.

- (1897), Die Lehre von der cardialen Dyspnoe und ihre historische Entwicklung, Wien. med. Wschr. 47 (1897) Nr. 27, 28.

Heller K. D. (1964), Ernst Mach. Wegbereiter der modernen Physik, Wien/New York.

Lesky ERNA (1965), Die Wiener medizinische Schule im 19. Jahrhundert, Studien zur Geschichte der Universität Wien, Band 6, Graz/Köln.

Mach Ernst (1910), Populärwissenschaftliche Vorlesungen, 4. Auflage, Leipzig.

- (1919), Die Leitgedanken meiner naturwissenschaftlichen Erkenntnislehre und ihre Aufnahme durch die Zeitgenossen, Leipzig.

Mises Richard von (1956), Positivism. A study in human understanding, New York.

Traube Ludwig (1846), Beiträge zur experimentellen Pathologie und Physiologie, Berlin.

- (1871), Gesammelte Beiträge zur Pathologie und Physiologie, Bände 1 und 2, Berlin, Band 3, herausgegeben von A. FraEnKel, Berlin 1878. 